\title{
CONTABILIDAD ESTRATÉGICA EN LA GESTIÓN DE EMPRESAS TEXTILES PERUANAS
}

\author{
STRATEGIC ACCOUNTING MANAGEMENT IN PERUVIAN BUSINESS TEXTILES
}

Raúl Alberto Arrarte Mera*

Docente Principal de la Facultad de Ciencias Contables

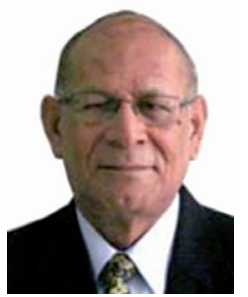

Universidad Nacional Mayor de San Marcos-UNMSM / Lima-Perú

[Recepción: Julio de 2013/ Conformidad: Agosto 2013]

\section{RESUMEN}

Esta investigación ha sido desarrollada el año 2012, con el apoyo económico del Consejo Superior de Investigaciones de la UNMSM de Lima-Perú, documental-explicativo-aplicativo con enfoque cualitativo, que se basa en el análisis de las empresas del Sector Textil de Confecciones Nacionales que listan en la Bolsa de Valores de Lima, comparadas con las empresas emblemáticas que lideran el mercado mundial. Nuestra industria textil, es de suma importancia, por ser un gran generador de divisas y empleo. Su contribución al PBI manufacturero se sitúa en el 11.6\%. Ocupa directamente 215,000 personas en tejido e hilandería y tiene el Cluster Textil de Confecciones más grande de Latinoamérica ${ }^{1}$.

La conclusión más importante es que el sector a futuro, deberá sustentar su idoneidad basado en la productividad y valor agregado de productos a base de algodón pima y alpaca, una ventaja competitiva aún sin competencia en el mundo.

Palabras Claves: Contabilidad estratégica, empresas textiles peruanas, empresas de clase mundial, Inditex. $\mathrm{H} \& \mathrm{M}$, Leonisa.

\begin{abstract}
This investigation made on the year 2012 with the help of the superior congress of investigation from UNMSM of Lima, Peru the explanatory documentary applicative with a qualitative approach is base of the analysis from the companies made from the national textile confections that are listed in the stock market of Lima which are compared with the emblematic companies that are in the top of the world market. Our textile industries are of great importance for been a generator of employments and foreign exchange. It's contribution to the PBI manufacturing is situated at $11.6 \%$ take directly 215,000 persons in the fabric and spinning mills also have a textile cluster in the confections of bigger textiles in Latin American.

The most important deduction is the future sector should support its suitability based on the productivity and the value-added products on the pima based of cotton and alpaca, having a competitive advantage even without competition in the world.
\end{abstract}

Keywords: Strategic accounting, Peruvian Textile Companies, world class companies, Inditex, H \& M. Leonisa.

* Doctor en Ciencias Contables y Empresariales. Maestro en Administración. Contador Público Colegiado. Docente Investigador de la UNMSM. E-mail: raularrarte@speedy.com.pe

1 http://www.produce.gob.pe/index.php/prensa/noticias-del-sector/280-destacan-importancia-del-sector-textil-confecciones - Visitado el $30 / 09 / 2012$ 


\section{INTRODUCCIÓN}

Una de las características de la industria textil peruana que la hacen competitiva internacionalmente, es el costo relativamente barato de su mano de obra calificada y no calificada. Por consiguiente, a futuro debe sustentar su competitividad basada en la productividad y el valor agregado de sus productos apoyados en el algodón y la alpaca. Así, los industriales tendrán mayores márgenes para combinar una adecuada remuneración con altos niveles de rentabilidad.

Un excelente trabajo nos informa al respecto. Según Angulo L, M. A. (2004, p. 146), "las mejores tecnologías en esta industria están orientadas al desarrollo de herramientas que permitan producciones más flexibles y enfocadas en la calidad del producto... hoy el avance tecnológico está proveyendo a los productores de prendas de vestir, la flexibilidad para ofrecer pequeñas órdenes de producción según los requerimientos del cliente y a precios competitivos".

\section{FUNDAMENTACIÓN DEL ESTUDIO}

Contabilidad estratégica, no es más que la búsqueda de un sistema de información integral, que incluye información financiera y no financiera para la toma de decisiones como soporte, lo que mantiene e incrementa la posición competitiva de una organización. Así, si la profesión contable establece este enfoque en todos sus clientes estaremos contribuyendo a su desarrollo integral y lograremos participar en la construcción de un país más competitivo.

¿Por qué elegimos el tema? Para responder a algunas de las siguientes interrogantes: ¿Es necesario tomar o no a un nuevo personal? ¿Qué productos exportar? ¿Qué mercados presentan las mejores creaciones para su comercialización? ¿Es necesario crear una oficina propia en el exterior? ¿Se debe nombrar un representante? La decisión estará en función al volumen de ventas que se proyecte desarrollar.

\section{OBJETIVOS}

\section{a) General}

Analizar si la contabilidad estratégica es importante en la gestión de empresas textiles peruanas.

\section{b) Específicos}

1. Identificar qué estrategias de precios se deben desarrollar para convertirse en empresas de clase mundial.

2. Definir qué herramientas de gestión se debe priorizar para ser más rentables y competitivas.

\section{FUNDAMENTACIÓN DEL ESTUDIO}

- Los resultados de esta investigación contribuirán a la difusión, desarrollo y concientización de la contabilidad estratégica y al perfeccionamiento profesional y tecnológico de los Contadores Públicos Peruanos que estudian en la Unidad de Posgrado de la Facultad de Ciencias Contables de la UNMSM en beneficio de la industria nacional para mejorar las estrategias de precios y herramientas de gestión.

- Permitirá que nuestra universidad forme excelentes profesionales con dominio de técnicas modernas validadas por el mercado global, que contribuirá a la acumulación social del conocimiento contableadministrativo.

- En un mercado de libre competencia, las empresas textiles peruanas deberán acostumbrarse a definir claras estrategias para alcanzar una ventaja competitiva. Esto asegurará calidad y buenos precios, lo que permitirá mejorar la rentabilidad y liquidez suficiente para facilitar su viabilidad en el largo plazo.

\section{MARCO TEÓRICO Y DEFINICIÓN DE CONCEPTOS}

\section{Contabilidad Estratégica}

Es la disciplina que provee de la información necesaria para formular y llevar a cabo las estrategias para alcanzar una ventaja competitiva. Es fundamental, para el proceso de toma de decisiones. Sirve para establecer los segmentos de mercados más rentables, definir y asignar los recursos necesarios, los planes de acción a ejecutar para sobresalir ante la competencia 
y la búsqueda de alianzas para permitirles ser más competitivos.

Por ello, para Gil, Jorge Manuel (4, 2012), gestionar es algo más que tomar decisiones en un sistema con un objetivo predeterminado. Implica acciones concretas que tienen contenido:

- Estratégico (vinculación con los escenarios futuros de la realidad probable y con las visiones de la organización a largo plazo).

- Táctico (adecuación de objetivos a condiciones de una realidad y mercado cambiantes).
- Operativo (elección de alternativas para solucionar los problemas de la realidad).

\section{Empresa Textil Peruana}

Las exportaciones de productos textiles y confecciones en setiembre de 2012 sumaron US\$ 183 millones, monto mayor en $8.5 \%$ respecto al mismo mes del año 2011, según informó el Banco Central de Reserva del Perú.

Veamos el siguiente cuadro de información actualizado:

\section{Cuadro $\mathrm{N}^{\circ} 1$}

\begin{tabular}{|c|c|c|c|c|c|c|}
\hline \multicolumn{7}{|c|}{ PRINCIPALES DESTINOS: EXPORTACIONES TEXTILES AÑO 2012} \\
\hline \multicolumn{7}{|c|}{$\begin{array}{l}\text { (MILLONES DE USS) } \\
\end{array}$} \\
\hline \multicolumn{4}{|c|}{ SETIEMBRE } & \multicolumn{3}{|c|}{ ENERO-SETIEMBRE } \\
\hline PAÍSES & 2011 & 2012 & Var \% & 2011 & 2012 & Var \% \\
\hline Venezuela & 41 & 53 & 31.4 & 208 & 502 & 140.9 \\
\hline Estados Unidos & 49 & 53 & 7.1 & 568 & 476 & $(16.2)$ \\
\hline Brasil & 11 & 12 & 13.3 & 76 & 76 & 0.0 \\
\hline Colombia & 11 & 8 & $(25.0)$ & 78 & 70 & $(10.6)$ \\
\hline Ecuador & 6 & 11 & 87.0 & 57 & 69 & 20.8 \\
\hline Chile & 7 & 7 & $(1.9)$ & 57 & 57 & $(0.3)$ \\
\hline Italia & 8 & 5 & $(38.0)$ & 56 & 43 & (22.9) \\
\hline Argentina & 5 & 5 & $(12.8)$ & 41 & 36 & (11.4) \\
\hline Bolivia & 3 & 4 & 33.6 & 27 & 35 & 28.8 \\
\hline Alemania & 4 & 3 & $(27.8)$ & 30 & 26 & $(13.2)$ \\
\hline México & 2 & 3 & 25.8 & 18 & 24 & 35.0 \\
\hline China & 1 & 2 & 49.5 & 16 & 18 & 8.8 \\
\hline Reino Unido & 3 & 1 & $(46.5)$ & 25 & 17 & $(30.4)$ \\
\hline Canadá & 2 & 3 & 31.0 & 15 & 16 & 9.2 \\
\hline Japón & 1 & 2 & 40.6 & 13 & 14 & 5.6 \\
\hline Resto & 15 & 12 & $(18.1)$ & 114 & 111 & $(2.8)$ \\
\hline TOTAL & 169 & 183 & 8.5 & 1,400 & 1,591 & 13.6 \\
\hline Norteamérica & 53 & 58 & 8.8 & 601 & 517 & $(14.0)$ \\
\hline Países Andinos 1/ & 67 & 83 & 23.8 & 428 & 733 & 71.2 \\
\hline Unión Europea & 20 & 13 & $(34.8)$ & 152 & 119 & $(21.7)$ \\
\hline Mercosur 2/ & 17 & 17 & 2.6 & 123 & 119 & $(3.1)$ \\
\hline Asia & 5 & 5 & 3.8 & 54 & 60 & 9.9 \\
\hline
\end{tabular}

1/Bolivia, Chile. Ecuador y Colombia.

2/ Brasil, Argentina, Uruguay y Paraguay

Fuente: SUNAT. Superintendencia Nacional de Administración Tributaria. Perú.

Como se observa en el periodo enero-setiembre, las exportaciones del sector textil sumaron 1,591 millones de dólares, $13.6 \%$ superior a las alcanzadas en el mismo periodo del año anterior, elaboradas en base a datos de la SUNAT. 


\section{Modelo peruano de desarrollo textil}

Un botón como muestra. Textil del Valle es una empresa de confecciones de prendas de punto, básicamente, de algodón Pima, cuya cultura organizacional se plasma en el siguiente párrafo: "Trabajamos como contract manufacturer, es decir, producimos prendas a pedido para las principales marcas del mundo.

Nosotros elaboramos básicamente prendas de vestir de tejidos de punto, polos con cuello, vestidos, sweatshirts, entre otros. Hacemos prendas de moda, no productos básicos; estos varían en colores, diseños, telas, temporada por temporada. Nuestros pedidos cambian constantemente tanto en volúmenes como en diseños. Tenemos clientes que trabajan hasta seis temporadas al año y en cada temporada los estilos que les fabricamos son diferentes.

Actualmente, el concepto país ya no existe ni en el origen ni en el destino; ahora son las marcas y las fábricas productoras. Nosotros hacemos prendas para Ralph Lauren, Lacoste, Náutica, Chicos, Swiss Army, entre otras firmas. Estas son marcas de primer orden y son ellos quienes nos indican los destinos de las prendas. Nosotros hacemos un contrato con ellos en el que se especifica cuántas prendas se deben incluir y su destino, el cual puede ser Asia, Europa, EE.UU., o América Latina. Aproximadamente el $80 \%$ de las prendas va a EE.UU., y el $20 \%$ restante va a diferentes destinos".

\section{Nuestra posición respecto al tema}

En esta entrevista advertimos, cómo la empresa peruana, se ha "identificado con la globalización". Fenómeno al que tenemos que adaptarnos, donde nunca como ahora, la comunidad internacional ha estado tan interrelacionada y comunicada a un número cada vez mayor de países, a la consolidación internacional de gustos y tendencias más uniformes. Cada vez es mayor el número de empresas transnacionales que operan en muchos países, según Navarro G, M $(2008,32)$ siguiendo criterios de estandarización o de adaptación al mercado y con ello, al medio sociocultural del país en el que están presentes.

Si comparamos la figura, salvando distancias, del valor que se paga por un kg., de papa blanca en el campo a $\mathrm{S} / .0 .50$ y el que se comercializa en el $\mathrm{Su}$ permercado a $S / .2 .50$. Es lo mismo a 5 veces su valor de origen. Hagamos pues, que nuestros empresarios textiles desarrollen la estrategia de "Ganar-Ganar", participando, proporcionalmente de las utilidades del distribuidor, beneficiando de paso al trabajador textil y al erario nacional. Para alcanzarlo necesitamos integrar la cooperación Empresa-Universidad-Estado. Sectores que aún, en pleno tercer milenio, no logran integrarse en el Perú.

Como es de notar, pese al pujante desarrollo del Sector Textil Confecciones nos falta un elemento fundamental para figurar en el Index mundial: desarrollar nuestras propias marcas. Habilidad tenemos, creatividad abundante, materia prima de primera calidad, mano de obra cuantiosa. Necesitamos decisión: pensar en grande para cosechar en grande.

El gurú John Shank, creador de la administración estratégica de costos, nos recomienda:

"No se empieza primero con el costo y después se obtiene el precio, sino que se comienza con el precio y luego se obtiene el costo". Para ello, necesitamos del apoyo de la contabilidad estratégica:

a) Identificar Cadena de Valor del negocio.

b) Practicar Benchmarking con el líder mundial.

c) Analizar los elementos que llevan al líder al éxito.

Veamos lo que nos dice Noboa, Fabrizio $(2005,1)$, al respecto:

"Si queremos mejorar nuestro posicionamiento estratégico, simplemente debemos comprobar si somos capaces de hacer alguna de las tareas que forman la cadena de valor en la provisión del bien o servicio, mejor que nuestros rivales (a través de la diferenciación) o más barato que ellos (reducción de costos)." En otras palabras, una empresa de sólido posicionamiento estratégico es líder en costos o es diferente al resto ( $y$ es capaz de cobrar una prima por ello)".

"Ser el líder en costos no es tarea fácil y depende en gran medida de las condiciones del sector. En algunas áreas es más fácil alcanzar economías de escala o acceso preferencial a materias primas, caminos tradicionales para reducir costos. Por ejemplo, hace años la producción en serie del Ford $T$ fue lo que le concedió ventaja frente al resto de competidores al reducir costos a través de economías de escala.. 
Ser líder en costos es conveniente para nuestro posicionamiento estratégico sólo cuando la empresa que alcanza esos niveles puede cobrar un precio cercano al promedio en el sector. En ocasiones, algunas empresas reducen costos a base de reducir la calidad de sus productos, lo cual amenaza su posibilidad de cobrar precios similares a la media del mercado, atentando contra su posicionamiento estratégico. Dado que el desarrollo tecnológico es un motor importante para la reducción de costos, las empresas nacionales no deben tener miedo a invertir en tecnología: en el mediano y largo plazo, el resultado será niveles inferiores de costos con iguales - 0 superiores!- niveles de calidad".

Otra alternativa para fortalecer nuestro posicionamiento estratégico es siendo diferente al resto. Bajo esta estrategia, las empresas buscan ser únicas a lo largo de alguna dimensión que los compradores perciben como importante. Si la empresa puede cobrar una prima por esa fuente de diferenciación, superior a los costos en los que incurre para entregar esa diferenciación, entonces fortalece su posicionamiento estratégico.

Las fuentes de diferenciación también dependen de la estructura del sector. Mientras la industria textil ofrece innumerables posibilidades de brindar un producto diferente y servir en un nicho de mercado único. Cabe resaltar que toda diferenciación depende de la capacidad innovadora de la empresa porque la diferenciación no debe estar ligada, exclusivamente, al producto, lo que puede darse en el sistema de entrega, en el servicio postventa, en el mercadeo, en el mismo cliente final, etc.

Estratégicamente, no es posible pretender atender todas las necesidades de un mercado.

\section{APLICACIÓN PRÁCTICA DE LOS RESULTA- DOS DE LA INVESTIGACIÓN}

El trabajo desarrollado sirve para mostrar a la Industria Nacional Peruana; en particular, al Sector Textil-Confecciones, la importancia de la contabilidad estratégica y su aplicación a la vida real. La necesidad de informarse de lo que hacen las empresas líderes para mantenerse en el mercado. De la informa- ción obtenida se desprende los siguientes resultados de nuestra investigación:

\section{Perú es productor del algodón Pima y Tanguis,} materias primas necesarias para la manufactura de determinadas prendas textiles y prendas de vestir, con particularidades únicas que posibilitan productos de elevada calidad dirigidos al segmento medio-alto del mercado. Sin embargo, la tendencia a futuro es la reducción paulatina de este insumo sustituida por la fibra sintética como el Poliéster. Aunque en el mundo, la producción sigue superando a la demanda, muchos detallistas y marcas conocidas están introduciendo prendas de algodón orgánico. La demanda es muy atractiva porque está en crecimiento, la competencia es menor y los precios suelen ser más altos porque se protege el medio ambiente, necesita de ese marco, es el quinto productor de algo más de 2,000 toneladas anuales de fibra extra larga. Por lo tanto, en el mediano plazo conviene al país incrementar su producción del algodón orgánico. Por esta razón se necesita un acercamiento del Ministerio de Agricultura con los productores de este insumo coordinado con los fabricantes de prendas de vestir.

\section{En los países andinos se produce la fibra de} Alpaca, única originaria de los Andes (Perú, Chile y Bolivia). En sus dos variantes: Alpaca Huancayo y Alpaca Suri. Fibra muy suave, con aspecto sedoso y propiedades térmicas únicas siendo el principal productor en el mundo; Australia hace grandes inversiones en programas de investigación desde 2003 respecto a: incremento del número de auquénidos, mejora de la genética, mejora de la productividad, apoyo en su investigación y desarrollo, promocionar la imagen de país como productor de fibras nacionales. En razón de lo expuesto, las Facultades de Biología de todo el Perú, deberían realizar permanentemente trabajos de investigación, con el auspicio de la industria textil, para mantener su ventaja competitiva a base de esta materia prima. Es importante, la cooperación Empresa-UniversidadEstado a corto, mediano y largo plazo. 


\section{Empresas como el Grupo Mango, son líderes en textiles gracias al feliz empleo de la estrategia de la Responsabilidad Social Empresarial (RSE).}

¿Qué hace la diferencia con las otras empresas de la competencia como Leonisa y Zara?.La práctica de la RSE, que se apoya en los valores fundamentales de la empresa: armonía, (opuesto al conflicto), humildad (opuesto a la prepotencia) y afecto (opuesto a la indiferencia). Estos valores iniciales se definieron a principios de los años 90 y posteriormente, se incorporaron en el Código de Ética del Grupo Mango, que constituye el marco de referencia de RSE, ya que la empresa está convencida que el comportamiento ético y sostenible de una organización es consecuencia de la calidad humana de las personas que la forman.

\section{CONCLUSIONES}

1. Resulta de particular importancia aplicar la contabilidad estratégica en la gestión de las empresas textiles peruanas, porque merced a ella, nos permite identificar la cadena de valor del negocio; practicar benchmarking con el líder mundial y analizar los elementos que le llevan a liderar el negocio textil al éxito. No es posible atender todas las necesidades de un mercado, pero sí desarrollar aquello que nos es permitido como país para explotar al máximo nuestras ventajas comparativas: algodón pima y lana de alpaca. Desplegar nuestras ventajas competitivas con instrumentos en materia de desgravación, como la Decisión 414 de la Comunidad Andina de Naciones (CAN), mediante la cual se libera de gravamen a una serie de productos, incluidos textiles, en el comercio entre el Perú y los demás países miembros de la CAN. Lo que facilitará el fomento de la exportación y de las cadenas productivas. El Ministerio de Producción del Perú, dentro de su Plan Estratégico Multianual tiene programas para promover la innovación de transferencia de tecnología.

2. Una empresa de clase mundial es aquella que puede competir en términos de eficiencia y calidad con cualquier otra en el globo. Para ello, se tiene que:
- Fijar los precios combinando el método de costo meta con el margen deseado.

- Cumplir normas internacionales de calidad.

- Utilizar estrategias de manufactura de punta.

- Practicar una correcta planificación del producto.

- Conservar un buen nivel de solvencia.

- Manejar un horizonte de deuda relativamente bajo.

- Desarrollar los nuevos postulados contables promovido por las Normas Internacionales de Información Financiera.

3. Entre las herramientas de gestión a priorizar bajo el enfoque de la contabilidad estratégica es importante aplicar:

- Opciones más económicas mediante previa auditoría energética: gas o electricidad.

- Limitar el riesgo comercial por concentración de ventas a un máximo de $20 \%$ por Grupo Empresarial.

- Tener marca propia con presencia local y en países vecinos para incrementar las ventas.

- Aprovechar las certificaciones ISO 9001-2000 para penetrar al mercado de la Unión Europea de manera agresiva y contribuir con la meta exportaciones para los próximos tres años.

- Fortalecer y renovar el Departamento de Diseño de Productos de manera permanente para hacer frente a la demanda personalizada y a las tendencias cambiantes de la moda.

- Implantar el sistema de costeo ABC para el control detallado de los costos y su reporte en tiempo real recordando que "información es poder".

- Mejoramiento continuo y transferencia tecnológica oportuna para agregar valor a los productos.

- Optimizar el 100\% de la capacidad instalada en productos con mayor valor agregado.

- Aplicar benchmarking estratégico en forma cotidiana, utilizando adecuadamente tecnologías avanzadas de manufactura a fin de cumplir con los objetivos de productividad. 


\section{RECOMENDACIONES}

1. Para desarrollar las ventajas que proporciona la Contabilidad Estratégica, corresponde a nuestras universidades nacionales promover el conocimiento de estas técnicas antes descritas, a través de sus Unidades de Posgrado así como, por intermedio de acuerdos entre la EmpresaUniversidad-Estado. Ya que existen intereses comunes que benefician a las entidades en juego. Fortalece las tecnologías existentes, y promueve e incorpora nuevas métodos de aplicación.

2. Es necesario que nuestro gobierno defina una clara política de apoyo a la industria textil basada en la lana de alpaca como materia prima. Con publicidad y posicionamiento en la mente de los consumidores del mundo, cada vez que se organicen Encuentros Internacionales en nuestro país y, a donde se localice la representación del Perú en el exterior. Propiciando la formación de Centros de Estudios Multidisciplinarios, respetando las funciones profesionales de sus integrantes orientados a promover las bondades de la lana de Alpaca.

3. Para facilitar la aplicación de las herramientas de gestión que practica la contabilidad estratégica, hay que restituir el crédito tributario en toda inversión orientada a la investigación universitaria estatal. Establecer los mecanismos de acercamiento y cooperación entre entidades nacionales e internacionales para conseguir la ayuda técnico-financiera paritaria a cargo de los organismos locales respectivos, orientado a promover permanentemente el consumo de la fibra de Alpaca.

\section{REFERENCIAS BIBLIOGRÁFICAS}

1. BRICEÑO. R. (2012) Comité Textil Sociedad Nacional de Industrias. Lima. Perú.

2. ESPEJO, Karen. (2011). La equidad con el personal es la fuerza interna de una compañía. Diario “Gestión”. Lima. Perú.

3. GIL, Jorge. (2012) Interdependencias entre la contabilidad de gestión y el capital intelectual: una visión metodológica, en clave latinoamericana. Universidad de la Patagonia. Argentina.

4. GOVINDARAJAN Vijay. SHANKJohn (1995). Gerencia Estratégica de Costos. Grupo Editorial Norma. Colombia.

5. NAVARRO, Fernando. (2008) Responsabilidad Social Corporativa. Editorial ESIC. Madrid. España.

\section{REFERENCIAS HEMEROGRÁFICAS}

1. ANGULO L. Miguel. (2004). Análisis del Cluster Textil en el Perú. Facultad de Ingeniería Industrial. Universidad Nacional Mayor de San Marcos. Lima. Perú.

2. NOBOA, Fabrizio. (2005). Mejorando el Posicionamiento Estratégico. Cámara de Industrias de Guayaquil. Revista Industrias, Año XI, No. 4 Guayaquil-Ecuador. 